\title{
Case 1 - Long-term response to bevacizumab and paclitaxel as second-line therapy in metastatic vaginal carcinoma
}

\author{
Sebastian Schroecksnadel ${ }^{1}$, Jasmina Suljkanovic ${ }^{1}$, Christian Marth ${ }^{1}$ \\ Commentaries: Antonio González-Martín and Domenica Lorusso
}

\begin{abstract}
The role of chemotherapy in the treatment of vaginal carcinoma has not been well studied and is controversial. This case report describes a 59-year-old patient diagnosed with vaginal squamous cell carcinoma in May 2008. Initial surgery was performed with subsequent radiation therapy; 3 months later histologically-confirmed pulmonary metastases were detected. Chemotherapy with cisplatin and topotecan was administered and resulted in a mixed-disease response. Therefore, chemotherapy was switched to weekly paclitaxel and bevacizumab, which induced a complete response. After more than 70 months of follow-up there has been no sign of recurrence and the patient shows no evidence of disease. This patient had an exceptional response to the combination of bevacizumab and paclitaxel, and this combination warrants further investigation for its potential in vaginal carcinoma.
\end{abstract}

Key words: bevacizumab, squamous cell carcinoma, vaginal carcinoma

\section{Introduction}

Vaginal carcinoma is a rare condition in postmenopausal women and accounts for less than $2 \%$ of all gynecological malignancies. The incidence of invasive vaginal cancer is $0.42-0.69$ cases per 100,000 women and has its age peak in the sixth and seventh decades. The vast majority of vaginal carcinomas are of squamous cell histology; adenocarcinomas account for only about $5 \%$ and other histological types are infrequent. Human papilloma virus (HPV) infection, especially HPV 16, as well as prior hysterectomy, genital warts, low socioeconomic status, chronic irritant vaginitis, and smoking have been shown to be risk factors for the development of vaginal squamous cell carcinoma [1-3].

Tumor stage is the most important prognostic factor for overall survival (OS) and metastasis-free survival in patients with vaginal cancer. In advanced FIGO stages III and IV, the risk of death is 4.7 -fold higher than for FIGO

'Department of Obstetrics and Gynaecology, Medical University of Innsbruck, Austria.

Correspondence to: Christian Marth, MD, PhD,

Department of Obstetrics and Gynaecology, Medical University, Anichstrasse 35 A-6020 Innsbruck, Austria.

Phone: +43 51250423051 - Fax: +43 51250423055

E-mail: christian.marth@i-med.ac.at

CANCER BREAKING NEWS 2016;4(1):36-40

DOI: $10.19156 / \mathrm{cbn} .2016 .0007$ stage I [4]. Five-year survival rates appear to be related to disease stage and have been reported to be $73 \%, 58 \%$, and $36 \%$ for patients with FIGO stage I, II, or III+IV disease, respectively [5].

Treatment of primary invasive vaginal cancer should be adapted for disease stage and vaginal involvement. Radiation and surgery are the current standard therapies. At present, surgery is largely restricted to the early stages of disease, and is performed as wide local excision, or simple or radical vaginectomy depending on tumor size. Lesions with a higher risk of recurrence in the lower third of the vagina require vulvo-vaginectomy in addition to inguinofemoral node dissection. Lesions in the upper thirds of the vagina require radical hysterectomy, including partial or total colpectomy with removal of paracolpic tissue and pelvic lymph nodes.

The main pillar of vaginal carcinoma treatment is radiation. In early disease stages, radiation therapy can be administered in addition to surgery with a curative intent. In higher stages, a combination of external beam radiotherapy and brachytherapy with total doses between 70 and $85 \mathrm{~Gy}$ depending on the location and extent of the tumor is used. The clinical target volume should include the vagina, the para-vaginal area up to the pelvic sidewalls and the bilateral pelvic lymph nodes.

Chemotherapy has played a relatively minor role in the management of vaginal carcinoma to date and has been 
used mainly for metastatic tumors. Concurrent chemotherapy in combination with radiation has been increasingly administered in patients with advanced disease [2, 3]. The most frequently used chemotherapeutic regimes include cisplatin, 5-fluorouracil, bleomycin and mitomycinC. However, the prognosis of recurrent disease is poor. In patients with distant metastasis, platinum-based chemotherapy (as applied in the treatment of cervical cancer) is widely used. There is currently no published evidence for significant efficacy with any second-line chemotherapy options in vaginal carcinoma.

\section{Case report}

A 59-year-old patient was referred to our unit in May 2008 with confirmed lymph node metastasis from an unknown squamous carcinoma in the right inguinal region. Computed tomography (CT) scanning showed a $7 \times 6 \mathrm{~cm}$ mass in the right inguinal region, and a CT-guided biopsy was performed. The patient had no previous gynecological or family histories, but did have documented chronic obstructive lung disease and had experienced one prior acute coronary syndrome event.
Gynecological examination showed a tumor of about $1 \mathrm{~cm}$ in diameter located in the distal vagina under the urethra. Transvaginal pelvic ultrasound, pap smear and tumor markers were all normal. The tumor biopsy revealed a partially keratinizing squamous vaginal carcinoma. No other metastases were detected. The tumor was therefore classified as FIGO stage III.

The patient initially underwent surgery with bilateral inguino-femoral lymphadenectomy, anterior hemivulvectomy with partial resection of the anterior vaginal wall and resection of the distal urethra.

Histological examination showed that the tumor reached to the resection margin on one side. A total of 28 lymph nodes were removed; five of the ten nodes on the right side showed tumor infiltration, including the large $7 \mathrm{~cm}$ node, whereas all 18 nodes on the left side were free of cancer. Subsequently, the patient underwent adjuvant external beam radiotherapy of the pelvis and inguinal regions, with a total dose of 50.4 Gy. At 3-month follow-up in September 2008 there was no sign of local recurrence. However, the presence of pulmonary symptoms triggered an x-ray and CT scan of the chest. Multiple pulmonary nodules (Figure 1)

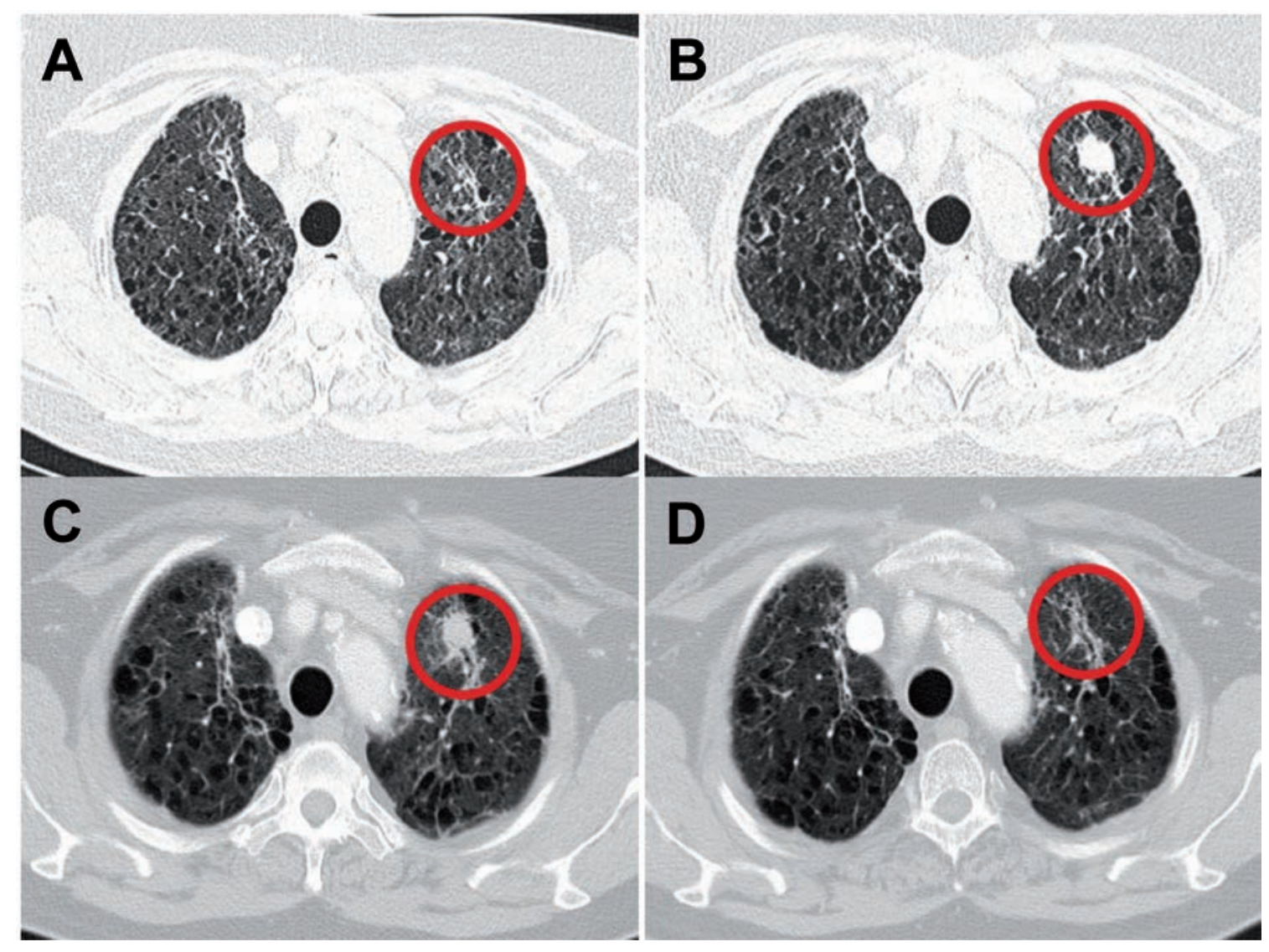

Fig. 1. Chest computed tomography (CT) scans at date of primary diagnosis (A), detection of pulmonary metastasis after three months, which was confirmed by CT-guided biopsy (B), after treatment with cisplatin/topotecan (C) and after treatment with paclitaxel/bevacizumab (D). 
were detected and a CT-guided biopsy was performed. Histology confirmed metastasis of a partially keratinizing squamous carcinoma.

Chemotherapy with cisplatin and topotecan was initiated and a mixed response was observed after six cycles (February 2009). In the absence of established alternatives, therapy was switched to weekly paclitaxel $\left(80 \mathrm{mg} / \mathrm{m}^{2}\right)$ and bevacizumab (10 mg/kg every two weeks). A complete response was documented after three cycles (June 2009) and therapy with paclitaxel and bevacizumab was continued for two additional months. The patient experienced several minor, low-grade hematological adverse effects (anemia, thrombocytopenia) and one event of febrile neutropenia with Escherichia coli sepsis and erysipelas of the right leg in June 2009, which was treated according to local guidelines. One dose of bevacizumab had to be skipped due to hypertension during treatment. Therapy with paclitaxel and bevacizumab was stopped after $5 \mathrm{cy}$ cles in August 2009 due to the pronounced recurrence of the erysipelas of the right leg.

The patient was followed up for six years after the end of treatment according to local clinical practice, including CT scans, and no progression has been detected to date. The patient is in good clinical condition, with urinary incontinence as the only late sequelae.

\section{Conclusion}

Due to the low incidence of vaginal carcinoma, no phase III clinical trials have been conducted and current treatment and guidelines are primarily based on retrospective

\section{References}

1. Hiniker SM, Roux A, Murphy JD, et al. Primary squamous cell carcinoma of the vagina: prognostic factors, treatment patterns, and outcomes. Gynecol Oncol 2013;131(2):380-5.

2. Di Donato V, Bellati F, Fischetti M, et al. Vaginal cancer. Crit Rev Oncol Hematol 2012;81(3):286-95.

3. Gadducci A, Fabrini MG, Lanfredini N, et al. Squamous cell carcinoma of the vagina: natural history, treatment modalities and prognostic factors. Crit Rev Oncol Hematol 2015;93(3):211-24.

4. Shah CA, Goff BA, Lowe K, et al. Factors affecting risk of mortality in women with vaginal cancer. Obstet Gynecol 2009;113(5):1038-45.

5. Creasman WT, Phillips JL, Menck HR. The National Cancer Data Base report on cancer of the vagina. Cancer 1998;83(5):1033-40.

6. Peters WA 3rd, Kumar NB, Morley GW. Carcinoma of the vagina. Factors influencing treatment outcome. Cancer 1985;55(4):892-7.

7. Thigpen JT, Blessing JA, Homesley HD, et al. Phase II trial of cisplatin in advanced or recurrent cancer of the studies and/or strategies used in cervical cancer. Surgery and radiation therapy are the current mainstays of therapy for local disease. Treatment of advanced and metastatic vaginal cancer with chemotherapy has not shown convincing efficacy [6, 7]. However, the combination of radiation and concurrent chemotherapy has shown promising results in patients with locally advanced cancer in terms of loco-regional control rate, disease-free and overall survival [8-11]. Several cases of prolonged long-term survival in progressed disease stages have been reported [12-14].

Nevertheless, chemotherapeutic strategies have largely proven ineffective for the treatment of recurrent vaginal carcinoma, and no effective second-line therapy has yet been identified. In this case, therapy of recurrent squamous cell carcinoma of the vagina with paclitaxel and bevacizumab was very effective, leading to complete remission and prolonged disease-free survival. Therefore, this combination may represent a potent alternative as second-line therapy for recurrent vaginal carcinoma.

\section{Acknowledgments}

The authors thank Nicola Ryan, an independent medical writer, who provided native English editing and journal styling on behalf of HPS. This editorial assistance was funded by PharmaMar, Spain.

\section{Conflicts of Interest}

The Authors declare there are no conflicts of interest in relation to this article.

vagina: a Gynecologic Oncology Group Study. Gynecol Oncol 1986;23(1):101-4.

8. Dalrymple JL, Russell AH, Lee SW, et al. Chemoradiation for primary invasive squamous carcinoma of the vagina. Int J Gynecol Cancer 2004;14(1):110-7.

9. Samant R, Lau B, E C, et al. Primary vaginal cancer treated with concurrent chemoradiation using Cis-platinum. Int J Radiat Oncol Biol Phys 2007;69(3):746-50.

10. Nashiro T, Yagi C, Hirakawa M, et al. Concurrent chemoradiation for locally advanced squamous cell carcinoma of the vagina: case series and literature review. Int J Clin Oncol 2008;13(4):335-9.

11. Dimopoulos JC, Schmid MP, Fidarova E, et al. Treatment of locally advanced vaginal cancer with radiochemotherapy and magnetic resonance image-guided adaptive brachytherapy: dose-volume parameters and first clinical results. Int J Radiat Oncol Biol Phys 2012;82(5):1880-8.

12. Rajagopalan MS, Xu KM, Lin JF, et al. Adoption and impact of concurrent chemoradiation therapy for vaginal cancer: a National Cancer Data Base (NCDB) study. Gynecol Oncol 2014;135(3):495-502. 
13. Kim JY, Lee KA, Kim BG, et al. Vaginal cancer with multiple liver and pulmonary metastases that achieved long-term survival. Obstet Gynecol Sci 2013;56(6): 416-9.
14. Holleboom CA, Kock HC, Nijs AM, et al. cis-Diaminechloroplatinum in the treatment of advanced primary squamous cell carcinoma of the vaginal wall: a case report. Gynecol Oncol 1987;27(1):110-5.

\section{Commentary 1}

Primary vaginal carcinoma is a very rare disease, for which clinical trial-based evidence for therapy is almost absent. This has resulted in a wide variety of treatment strategies being used in women with vaginal carcinoma. In addition, because vaginal carcinoma is rare, treatment approaches have been extrapolated from other diseases that might have some common etiopathogenic factors. In particular, because the majority of vaginal cancers are of the squamous subtype and HPV has been identified as a relevant causative agent, metastatic vaginal carcinoma has been treated with regimens that have shown some activity in cervical squamous cell carcinoma (SCC). Traditionally, platinum-based chemotherapy has been used to treat recurrent or metastatic vaginal cancer. However, data on responses are anecdotal and prognosis is very poor, with short overall survival. The treatment of the above case demonstrated these points because she was treated with the cisplatin-topotecan combination that was the standard of care for metastatic or recurrent cervical SCC in 2008. However, after 6 cycles of therapy the patient was switched to a combination of paclitaxel and bevacizumab due to the absence of an adequate response.

Paclitaxel has previously been shown to be active in combination with cisplatin in the neoadjuvant setting and in combination with carboplatin and concurrent external beam radiotherapy for the treatment of primary vaginal carcinoma [1, 2]. However, this is the first report on the activity of weekly paclitaxel in a patient with metastatic vaginal carcinoma previously treated with cisplatinbased chemotherapy.

Bevacizumab is a humanized monoclonal antibody that targets vascular endothelial growth factor and has an anti-angiogenic effect. Bevacizumab monotherapy has shown activity in patients with persistent or recurrent SCC of the cervix in a phase II study [3]. In that trial, 46 patients previously treated with one or two cytotoxic regimens for recurrent disease received bevacizumab monotherapy $15 \mathrm{mg} / \mathrm{kg}$ every 21 days; the response rate was 10.9\% (90\% confidence interval: 4\% to 22\%). A recently published phase III trial has demonstrated that adding bevacizumab to standard paclitaxel-cisplatin for the front-line therapy of recurrent or metastatic cervical cancer improves overall survival [4]. The patient reported in this case may be the first case of metastatic vaginal carcinoma treated with bevacizumab reported in the literature. The excellent response and outcome observed in this patient clearly demonstrate the activity of the bevacizumab and weekly paclitaxel combination in this orphan disease and merits further investigation in a prospective clinical trial.

Antonio González-Martín

Department of Medical Oncology MD Anderson Cancer Center, Madrid, Spain E-mail: agonzalezm@mdanderson.es

\section{References}

1. Benedetti Panici P, Bellati F, et al. Neoadjuvant chemotherapy followed by radical surgery in patients affected by vaginal carcinoma. Gynecol Oncol 2008;111(2):307-11.

2. Mabuchi S, Kawano M, Isohashi F, et al. First two cases of primary carcinoma of the vagina successfully treated with concurrent weekly carboplatin plus paclitaxel, external beam radiotherapy and high-dose-rate interstitial brachytherapy: a case report and published work review. J Obstet Gynaecol Res 2015;41(1):156-61.
3. Monk BJ, Sill MW, Burger RA, et al. Phase II trial of bevacizumab in the treatment of persistent or recurrent squamous cell carcinoma of the cervix: a Gynecologic Oncology Group study. J Clin Oncol 2009;27(7):1069-74.

4. Tewari KS, Sill MW, Long HJ 3rd, et al. Improved survival with bevacizumab in advanced cervical cancer. N Engl J Med 2014;370(8):734-43. 


\title{
Commentary 2
}

The authors report on a patient with a vaginal carcinoma progressing during first-line chemotherapy with cisplatin-topotecan. The patient was switched to second-line chemotherapy with weekly paclitaxel and bevacizumab, which was associated with an impressive response. The response to treatment is not surprising from my point of view. Squamous vaginal carcinoma is a rare disease and, given that there is therefore little available evidence about its treatment, management strategies should be derived from those for tumors with a similar histology located in other organs. Bevacizumab is the only agent reported to increase survival when used to treat advanced squamous cervical cancer, with which vaginal carcinoma shares not only its histology but also a causative agent in the human papilloma virus (HPV). Via its E6 protein, HPV stimulates vascular endothelial growth factor (VEGF) production, meaning that antiangiogenic agents are active against HPV-related disease. Moreover, weekly paclitaxel has antiangiogenic activity and studies in ovarian cancer report impressive synergy when bevacizumab and weekly paclitaxel are given together. The combination of weekly paclitaxel and bevacizumab merits further exploration in the treatment of HPV-related tumors, including squamous vaginal carcinoma.

\author{
Domenica Lorusso \\ Gynaecologic Oncology Unit \\ Fondazione IRCCS, Istituto Nazionale Tumori, Milano, Italy \\ E-mail: domenica.lorusso@istitutotumori.mi.it
}

